\section{An interesting case of inflammatory myofibroblastosis}

A twenty six year old female presented with intermittent, colicky abdominal pain associated with bilious vomiting of 1 week duration, and complete obstipation for one day. The patient also complained of loss of weight and appetite for 6 months. Laboratory findings were nonspecific. CT of the abdomen showed thickening of the splenic flexure and dilatation of proximal bowel loops. Colonoscopy showed an ulceroproliferative growth in the splenicflexure. Thescope could not be negotiated any further beyond this.
Colonoscopicbiopsy revealednonspecific inflammation. As the patienthad features of large bowel obstruction, the diagnosis of colonic malignancy was suspected. Intraoperatively, there was a circumferentialgrowth of size $4 \times 4 \mathrm{~cm}$ involving the splenic flexure resulting in closed loop obstruction with a dilated and edematous caecum, ascending colon and transverse colon.Due to doubtful viability of the colon, a subtotalcolectomy with ileo-rectal anastomosis was done.The histopathology showed myofibroblasticcells and mixed inflammatory infiltrate suggestive of inflammatory myofibroblastosis of thecolon. Immunohistochemistry (IHC) was positive for ALK, SMA,desmin.and negative for CD117. (Figure 1, a-d)
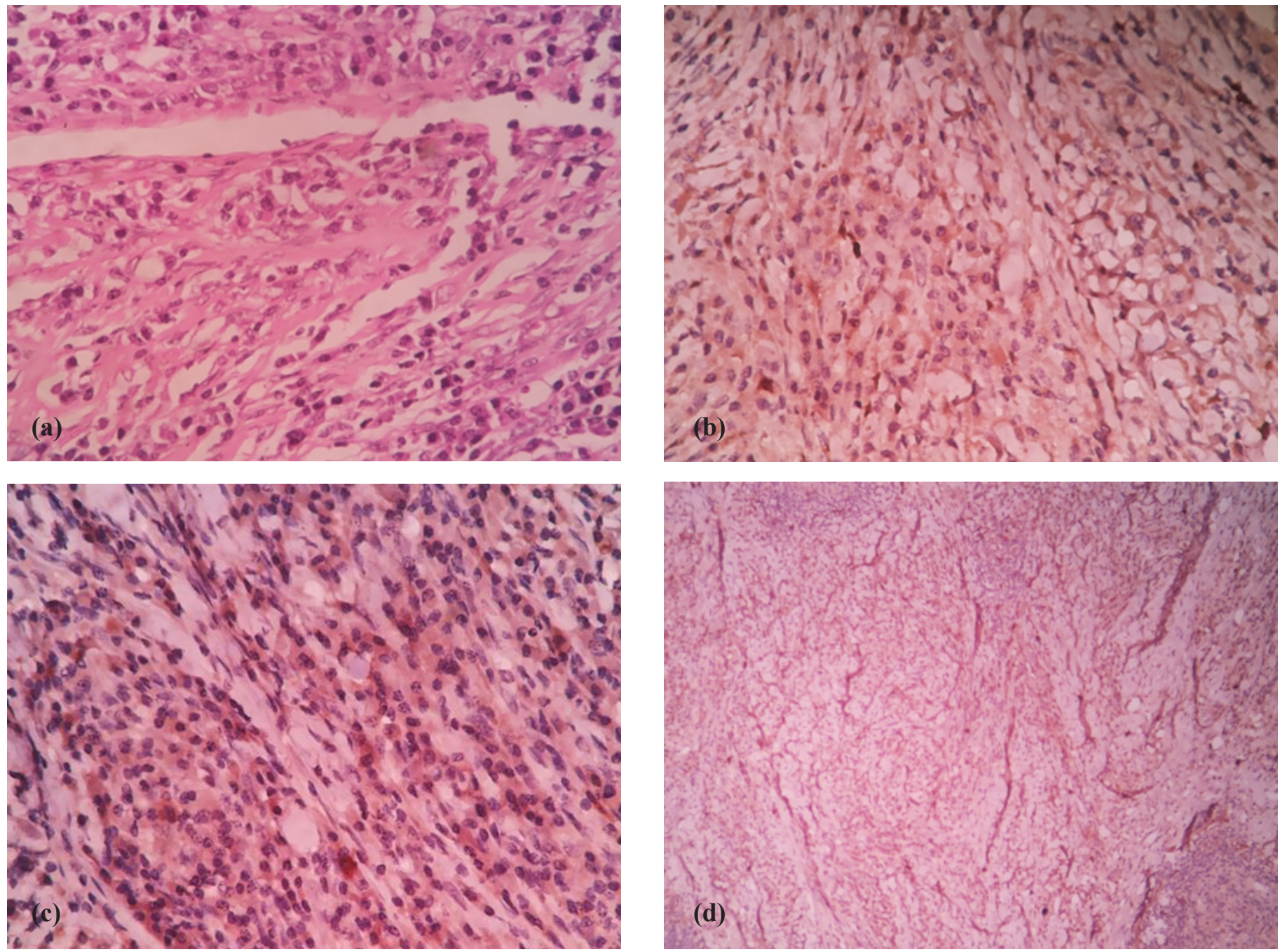

Figure 1: Histopathological specimen showing (a): characteristic myofibroblastic cells (b): IHC demonstrating ALK positivity (c): IHC demonstrating SMA positivity and (d): IHC demonstrating desmin positivity. 


\section{Discussion}

Since its first description in 1973, the evolution of Inflammatory MyofibroblasticTumour (IMFT), from a rare, unknown, reactive inflammatory process to a neoplasm of intermediate biologicalpotential has been remarkable.Though a plethora of synonyms like plasma cell granuloma, plasmacell pseudo-tumour, inflammatory myofibrohistiocytic proliferation,omental-mesenteric myxoid hamartoma and inflammatory pseudo-tumour (IPT) have been used to describe this rare entity, the term 'Inflammatorymyofibroblastictumour'(IMFT) ${ }^{1}$ is apt in paying credence toits characteristic myofibroblastic cell type. IMFTs occur in all age groups with no sexual predilection. ${ }^{1,2}$ Though its occurrence indiverse body sites is well documented, the common sites of involvement are the lung, mesentery, liver, and spleen.Intestinal involvement is however rare. Constitutional symptoms are quite infrequent. ${ }^{1,5}$ Laboratory abnormalities in IMFT are nonspecific, making the diagnosis difficult. ${ }^{1,5}$ The etiology of IMFT is not clear with diverse postulatesvarying from it being of neoplastic origin, to it being an immunological response to infection or inflammation.Overexpression of interleukin- 6 and cyclin D1, cytogenetic abnormalities, including ALKgene rearrangements on chromosome1,7,17,20,22, have also been described. ${ }^{1}$ Intestinal IMFTs are rarelyclearly delineated preoperatively on radiographic imaging ${ }^{1,3}$ due to clinical features of obstruction. Since the first case of colorectal IMFT was described in the rectum in 1995, there have been only about 25 cases of the same so far in literature. ${ }^{1,4}$ IMFTs in the colon and rectum present the same clinico-pathologicfeatures as observed in colorectal carcinoma, including altered bowelhabits, and are macroscopicallymostly ill-defined masses making endoscopic or radiological diagnosis difficult. ${ }^{1}$ Hence,immunohistochemistry is crucial to reaching an accurate diagnosis with the cells being characteristically positive for mesenchymal markers like vimentin but negative for CD117 and CD34. ${ }^{2}$ Completesurgical excision is the mainstay of treatmentand precludes recurrence. The prognosis of a colorectal IMT is more favourable, without any reported recurrence between 10 months and 4.5 years after surgical intervention. ${ }^{1,4}$ The role of agents like infliximab, adjunctive radiotherapy or chemotherapy is unclear. ${ }^{3,4}$

In conclusion, as IMFT encompasses a diverse spectrum of organ involvement with varied clinicalpresentations. Diagnosis rests on the histological hallmark of myofibroblastic proliferation and mixed inflammatory infiltrate. These lesions have an indolent course and are amenable to completesurgical excision. Thoughpre-operative diagnosis is bewilderingly difficult, a high index of clinical suspicion is required to recognise these rare lesions which frequently mimic colonic malignancy or tuberculosis, andcan present as a surgical emergency.

JMV AMARJOTHI A AMUDHAN R PRABHAKARAN D BENNET D KANNAN Department of Surgical Gastroenterology,
Madras Medical College,
Rajiv Gandhi Government General Hospital,
Chennai, Tamilnadu, India Correspondence: JMV Amarjothi Email:drmosesvikramamarjothi@hotmail.com

\section{References}

1. Simona Gurzu, Tivadar Bara, and IoanJung:Journal of Clinical Oncology, Vol 31, No 10 (April 1), 2013: pp e155-e158

2. Karnak I, Senocak ME, Ciftci AO, et al. Inflammatory myofibroblastictumour inchildren: Diagnosis and treatment. J PediatrSurg2001;36:908-912.

3. Bajaj P, Harris L, Vermillion JM, et al. An uncommon presentation of inflammatory myofibroblastictumour. Hosp Physician 2007;43:27-30.

4. Atilli SVS, Chandra CR, Hemant D, et al. Retroperitoneal inflammatory myofibroblastic tumour- a case report. World J Surg Oncol 2005;3:66.

5. Khoddami M, Sanae S, Nikkhoo B, et al. Rectal and appendiceal inflammatory myofibroblastictumours. Arch Iran Med 2006;9:277-281. 